\title{
Brachial plexus injury after median sternotomy
}

\author{
J G GR A H A M, I F P Y E, A N D I N F M C Q UE N \\ From the Departments of Neurology, University Hospital of Wales, Cardiff, and the \\ Leicester Royal Infirmary, Leicester
}

SUMMARY Five patients are described with brachial plexus injuries complicating median sternotomy for cardiac surgery. The lower roots (medial cord) of the plexus were most affected and in some cases the lesions were bilateral. Pain was a prominent feature and although motor and sensory symptoms both occurred, motor signs were more prominent than objective sensory loss. Recovery was protracted and sometimes incomplete. Traction or compression of the plexus (or both) are the major possible mechanisms of injury; our experience and that of others suggests that traction is the more probable mechanism.

Brachial plexus injuries have been recognised for many years as a complication of abdominal or thoracic surgery. ${ }^{12}$ Inappropriately sited shoulder braces may compress the brachial plexus and stretching can follow malpositioning of the patient on the operating table, particularly when muscle tone has been abolished by relaxant drugs. Care is taken now to avoid hyperabduction of the arms which is well known to subject the plexus to potentially dangerous traction. ${ }^{3}$ In the past 25 years widespread use of the median sternotomy incision has developed in cardiac surgery. Brachial plexus injury after median sternotomy has been recorded surprisingly infrequently and in limited detail. $^{4-8}$ Therefore, we wish to report our experience by describing the clinical details of five patients, since the condition may not always be recognised in cardiothoracic surgical units.

\section{Case reports}

Case 1 A 50-year-old male clerk (UHW 006047) suffered from severe angina and repeated episodes of acute coronary insufficiency following myocardial infarcts in 1966 and 1967. In December 1975 coronary artery endarterectomy and vein grafts were performed through a median sternotomy using cardiopulmonary bypass. Immediately after the operation the patient was aware of pain and numbness in the fourth and fifth digits and along the ulnar border of the left

Address for reprint requests: Dr IF Pye, The Leicester Royal Infirmary Leicester LE1 5 WW, England.

Accepted 14 May 1981 hand extending up the ulnar border of the forearm for five centimetres above the wrist. The pain gradually spread along the inner aspect of the arm to the axilla and adjacent chest wall with radiation over the shoulder into the neck. It was severe, uninfluenced by posture, and disturbed sleep. Motor function of the left hand was impaired with difficulty in clenching the fist. When examined one month after the operation, there was acute tenderness in the left supraclavicular fossa and movement of the left arm and shoulder provoked severe pain. There was early wasting with associated weakness of the intrinsic muscles of the left hand, but abductor pollicis brevis was relatively spared. The flexor and extensor muscles of the left fingers and wrist, the pronators of the left forearm and the left triceps were also weak. Although symptoms were absent on the right, the extensors of the fingers and the intrinsic hand muscles including abductor pollicis brevis were weak. Apart from reduction of the left triceps jerk the tendon reflexes in both arms were normal. Sensory impairment to pin prick and light touch involved the left fourth and fifth fingers, the ulnar border of the hand and medial half of the arm up to and including the apex of the axilla. Position and vibration sense were absent in the left fourth and fifth digits. There was no clinical or radiological evidence of a cervical rib. Nerve conduction studies demonstrated a normal median but a small amplitude ulnar sensory action potential with normal latencies for each. Motor conduction in the forearm segments of the left median and ulnar nerves was normal. The intracutaneous injection of $0.1 \mathrm{ml}$ $1: 1000$ histamine into each forearm produced a symmetrical flare reaction on both arms. One month later the pain was less severe and involved the left arm below the elbow with radiation into the hand and fourth and fifth digits. The power of flexion and ex- 
tension of the wrist had improved but the other weakness including that of the right hand was unchanged and the left triceps jerk was absent. All modalities of sensation were impaired in the left fourth and fifth fingers and pain loss extended along the ulnar border of the hand and forearm to just above the wrist. In the axilla itself, sensation to pin prick was lost. Gradually the neurological symptoms improved and five months after operation (April 1976) the pain had disappeared and the left hand was functionally normal. Unfortunately, the angina had recurred in March and as the patient was experiencing crescendo angina, coronary angiography was repeated in May. This showed an occlusion in the mid part of the anterior descending branch of the left coronary artery. In June 1976 a median sternotomy was performed and using cardio pulmonary bypass, a vein graft taken from the left arm was inserted successfully into the anterior descending coronary artery. The course after operation was satisfactory, but, since this second operation, the patient has noticed numbness of the left fourth and fifth fingers. Examination in November 1976 showed flattening of the inner aspect of the left forearm, and minimal weakness of abduction, adduction and extension of the left fingers, and of the left grip with otherwise normal power. The tendon reflexes were now normal. Sensation to pin prick was impaired in the ulnar distribution in the left hand with splitting of the fourth finger and additionally joint position sense and vibration sensation were defective in the fifth finger. Nerve conduction studies were repeated and the results were unchanged. The second operation relieved the patient's cardiac symptoms and he returned to full time work with no functional disability in the left hand.

Case 2 A 57-year-old miner (UHW 240893) had a right coronary endarterectomy and bilateral coronary artery vein grafts performed through a median sternotomy using cardio pulmonary bypass at the beginning of November 1976. He had little memory of the first five days after operation but on day six became aware of numbness and tingling in the left fourth and fifth fingers. Shortly after this, he developed continuous pain in the left forearm and this was sufficiently severe to disturb his sleep. He had no symptoms in the arm before the operation and there was no other relevant history. On examination three weeks after operation, he had a minimal left ptosis but no other features of a Horner's syndrome and the cranial nerves were normal. The significant neurological abnormalities were confined to the left arm where there was marked atrophy of the intrinsic hand muscles and wasting of the flexor and extensor forearm groups of muscles. There was wasting of the left triceps predominantly involving the long head. The supraspinati were wasted bilaterally, but in spite of these changes, which were more obvious on the left, power appeared good. The following movements were weak: extension of the elbow, supination and pronation of the forearm, extension of the wrist, flexion and extension of the fingers, and abduction and adduction of the fingers and thumb. The left triceps jerk was less brisk than the right but the biceps and supinator reflexes were bilaterally symmetrical and normal. Contact dysaesthesia, present over the tips of all the fingers, was more pronounced in the fourth and fifth digits. It extended up the inner border of the forearm to the elbow and sensation to pin prick was impaired in the same distribution. Joint position sensation was impaired in the left fifth finger and, to a lesser extent, in the fourth finger and two point discrimination was lost over the tip of the former. Vibration sensation was intact. Nerve conduction studies showed a small left radial nerve action potential, an absent left ulnar sensory action potential, a left median sensory action potential measuring $13 \mu \mathrm{V}$ in amplitude compared to $20 \mu \mathrm{V}$ on the unaffected right side, and motor conduction velocities in the left median and ulnar nerves which were just below the lower end of the normal range. Nearly four years later he has no motor dysfunction, but sensory loss persists in the $\mathrm{C} 8 \mathrm{der}$ matome and seems likely to be permanent.

Case 3 (UHW 195965) At the age of 16 years this boy presented with a "'flu" like illness followed by a pleural effusion. He appeared to recover but three weeks later developed cardiac tamponade; $180 \mathrm{ml}$ of haemorrhagic fluid was then aspirated from the pericardium. No responsible agent was identified. Four months later signs of constrictive pericarditis developed and seven months after his initial illness he was subjected to total pericardectomy through a median sternotomy. During operation he was supine with both arms by his sides. He was seen in the Neurology Department two weeks post operatively because he was thought to have developed an ulnar nerve lesion. The surgical notes recorded complaints of pain in the right hand one day after operation, but the patient did not recall symptoms until one week later. He then complained of intractable aching pain in the ulnar two fingers of the right hand and the ulnar aspect of the forearm as far as the elbow. The pain which greatly disturbed his sleep was uninfluenced by posture and was neither exacerbated nor relieved by movements of the neck, shoulder or elbow. Examination revealed minimal weakness of the long extensors of the fingers on the left side but no other abnormality here. There was weakness and slight wasting of the abductor pollicis brevis, the interossei and the flexors of the fingers on the right side, with impairment of superficial sensation in the $\mathrm{C} 8 \mathrm{der}$ matome. The reflexes were normal, there was no Horner's syndrome, and no other neurological abnormality. Vibration sense was lost on the right in digits four and five, and position sense in digit five. Severe and intractable pain continued for four months after the operation and then began to subside, disappearing altogether eight months after operation. Motor function started to improve two months after operation, abductor pollicis brevis being most severely affected. Sensory signs disappeared at four months apart from residual impairment of superficial sensation on the medial aspect of the forearm only. 
Nerve conduction studies performed one month after operation showed abnormalities of both median and ulnar sensory nerve action potentials but normal motor conduction. Radiographs of his neck were normal and did not show cervical ribs nor any abnormality of the transverse process of $\mathrm{C7}$.

Case 4 (LRI 64548) This 59-year-old building contractor with severe angina had a triple vein graft and coronary endarterectomy through a median sternotomy in 1977. During the operation the patient was supine with one pillow under the head, neck slightly flexed and arms by his sides. He had no clear recollection of the first two post operative days, but on the third day, when he was beginning to take notice of his surroundings, he became aware of numbness and tingling in the right fourth and fifth fingers and along the ulnar border of the right hand and forearm to the elbow. The right hand and arm were weak and clumsy and there was severe pain in both shoulders. Although aware that the left hand did not feel completely normal, he had not paid much attention to it because of the obvious disability on the right. He had suffered from mild maturity-onset diabetes since 1976 and this was controlled by dietary restriction and glibenclamide, but preoperatively there had been no neurological symptoms. On examination seventeen days after operation there was no Horner's syndrome and the cranial nerves were normal. The abnormal neurological signs were confined to the upper limbs. In the right hand wasting of the interossei, thenar and hypothenar eminences was associated with marked weakness (MRC grades two or three) and the flexors of the fingers showed a similar degree of weakness. The power of extension of the right fingers was MRC grade three and flexion and extension of the wrist and elbow were MRC grades three or four. There was minimal weakness of the right supraspinatus. In the left hand and arm there was no wasting but less severe weakness (MRC grade four) was present in the muscle groups listed above. The right triceps jerk was diminished, the other tendon reflexes being normal. Sensation to light touch and pin prick was impaired in the right fourth and fifth fingers and along the ulnar border of the hand and arm almost to the axilla. Joint position sense was lost in digit five and impaired in digit four, and vibration sense was defective but not absent in both these fingers. Sensation was normal in the left arm and hand. There was no visible bruising but palpation of the right axilla was very painful. No cervical rib or band was palpable and a cervical rib was not shown on radiographs. Nerve conduction studies at this time showed low amplitude median and absent ulnar sensory nerve action potentials bilaterally. The motor conduction velocity was $42.5 \mathrm{~m} / \mathrm{s}$ in the right ulnar nerve but motor conduction was normal in the left ulnar and both median nerves. Four months after operation the pain in the shoulders and symptoms in the left hand had resolved. He still had numbness and tingling in the right fourth and fifth fingers and along the ulnar border of the right hand which remained weak although power was improving. Seven months after operation the abnormal neurological signs were restricted to the right hand and arm: wasting of the intrinsic hand and flexor group of forearm muscles was associated with significant weakness (MRC four). Extension of the fingers and wrist and flexion and extension of the elbow had recovered almost full power. The tendon reflexes were normal. Sensation to light touch and pin prick remained impaired in digits four and five and along the ulnar border of the hand and arm to the elbow. Vibration and position sensation were still defective in the fourth and fifth fingers. Two years after operation the patient was reported to have a degree of residual weakness in the right hand.

Case 5 (LRI 9217) In September 1978 a 53-year-old hosiery knitter underwent a median sternotomy for replacement of his aortic and mitral valves by Bjork Shiley prostheses. He had little memory of the first two days after operation but on day three he became aware of severe aching pain in the left arm and inability to move the left hand properly. He complained of pins and needles in the left hand and also over the left side of the neck. The surgical notes recorded weakness and clumsiness of the left hand with impaired sensation over the left $\mathrm{C} 8$ dermatome. The pain in the arm was replaced after four weeks by a clamp like feeling which regressed over the next month so that only the arm above the elbow was affected. During this time function of the left hand improved gradually. Examination two months after operation showed wasting and weakness of the left supraspinatus, rhomboids, pectoralis major, flexor group of forearm muscles and abductor pollicis brevis. In addition the left infraspinatus, deltoid, biceps, interossei, abductor digiti minimi and opponens pollicis were weak. Contact dysaesthesia to light touch was present over the left side of the neck, the left arm and the upper chest in the C4-T3 distribution and appreciation of pin prick was impaired but not lost in the same area. Position sense was abnormal in the left fourth and fifth fingers but vibration sensation was intact. The tendon reflexes were normal, there was no Horner's syndrome or other neurological abnormality. The symptoms continued to improve and twelve months after the operation the patient had good function in the left arm and hand, although he said that the arm still felt heavy, and sensation to contact was peculiar over the left shoulder and side of the neck. There was no clinical or radiological evidence of a cervical rib.

\section{Discussion}

Five patients have been described in whom brachial plexus injuries followed median sternotomy. Intractable pain was a prominent feature in all patients. Motor and sensory abnormalities were seen and in the three patients with bilateral lesions both sides were not affected with equal 
severity. Four patients had sensory changes predominantly or entirely in the distribution of the lower roots of the brachial plexus. In the fifth patient, although sensory impairment was present in the dermatomes $\mathrm{C} 4$ through to $\mathrm{T} 3$, loss was maximal in the $\mathrm{C} 8 / \mathrm{T} 1,2$ distribution. In all five patients the main motor deficit affected muscles supplied by the lower roots of the plexus, constituting its medial cord. Additionally, two of the cases had unequivocal clinical evidence of less severe upper root (lateral cord) involvement. Although significant motor and sensory features were present in each patient, it was noticeable that in the less severely affected arm of those with bilateral lesions, motor deficit predominated over sensory loss. In five patients reported by Kirsh $e t a l^{5}$ details of symptoms are not stated but motor signs were more marked than sensory ones. An electromyographic study was performed by Honet et $a l^{8}$ on patients after median sternotomy for open heart surgery. Limited clinical information is given but their results suggested more severe motor than sensory involvement in both symptomatic and asymptomatic patients with the main impact on the medial cord of the plexus. None of the reported cases including our own showed any anatomical peculiarity such as cervical rib or abnormally long transverse processes of the lower cervical vertebrae. All our patients were male and we were interested to observe that all the cases reported by Kirsh et al and the majority in the series of Honet et al were also male. Although, it is possible to speculate about the reasons for this apparent male preponderance, no definite explanation is forthcoming.

Follow-up of our patients indicates that although motor and sensory improvement occur recovery may be protracted and incomplete. The least complete improvement occurred in the subject with diabetes (case 4) which may have made his nerve roots more vulnerable to the effects of trauma in the first place, in addition to impairing their eventual recovery. The patients reported by Kirsh et $a l^{5}$ made a full recovery and this contrasts with our observations. Although we found little serious residual functional disability, our experience suggests that the prognosis for complete recovery is not uniformly good, especially in the more severe brachial plexus palsies complicating median sternotomy.

The precise mechanism of injury is uncertain but traction of the plexus or compression between the clavicle and first rib during retraction of the sternal halves have been postulated. The plexus would be vulnerable to either process as its con- stituent nerve roots are fixed proximally at their points of exit from the vertebral canal and distally the nerves are tethered partially to the axillary fascia and also as they attach to supply individual arm muscles. A study was performed on a fresh cadaver (a 42-year-old female) prior to routine autopsy examination. The brachial plexus was displayed bilaterally. During the dissection pectoralis major was separated from the clavicle, reflected laterally and the clavicular part resected. Pectoralis minor was detached from the coracoid process, reflected medially and divided. The anterior fibres of deltoid were separated from the clavicle and removed. A $5 \mathrm{~cm}$ block was placed under the shoulders and the head rested on a sponge so that the neck was extended and the head was midline. This simulated the operative position. After median sternotomy, the halves of the sternum were separated by $14 \mathrm{~cm}$ using a retractor. This alone had no appreciable effect on the brachial plexus, but stretching of the trunks and cords occurred if the manoeuvre was combined with rotation of the head to the opposite side or abduction of the ipsilateral arm. The trunks, divisions and cords of the plexus were relaxed when the shoulder girdle was elevated as in shrugging the shoulders. No evidence of compression of the plexus between clavicle and first rib was noted in this artificial situation. Similar and in some cases more elaborate dissection studies by other workers have also suggested that traction and not compression is the most significant aetiological factor. ${ }^{3}{ }^{6} 9$ In a supine cadaver, abduction of the arms to $90^{\circ}$ or more, contralateral rotation of the head and abduction plus extension of the arms all increased tension on the plexus and these observations correlate with the development of palsies in patients subjected to operation in these positions. ${ }^{6}{ }^{9}$ Kirsh et al $^{5}$ stress the importance of upward rotation of the first rib in producing stretching of the brachial plexus as the sternal retractor is opened, and in their cases considerable left ventricular hypertrophy made adequate surgical exposure difficult so that wide separation of the sternal halves was needed. It would seem reasonable to postulate that the severity of traction and the duration of operation may combine to determine the degree of injury and whether the deficit will be permanent. Details of the operative position are unavailable in three of our patients, but the other two had their arms by their sides during operation so that even when the arms are adducted the plexus remains vulnerable to stretching or compression or both. In order to avoid excessive stretching of the contralateral plexus 
it appears to be important to ensure that the intubated patient's head is maintained in the mid line and not turned to one side.

Nerve conduction studies showed abnormalities in all three major forearm nerves in one of our patients but in general the greatest abnormality was found in the ulnar nerve with less in the median nerve and little, if any, in the radial nerve. The nerve conduction results were compatible with partial axonal degeneration at plexus level and certainly indicated more than a neurapraxia.

The detection of five cases of serious brachial plexus injury after median sternotomy in a period of less than three years suggests that this complication may not be uncommon. The predominance of sensory symptoms in the fourth and fifth digits gives the superficial impression of an ulnar nerve lesion and some cases may well pass unrecognised in this guise. In addition during the immediate period after operation, if patients have been given sedation and analgesics they may not complain of neurological symptoms. Neurological deficit can be overlooked relatively easily at this time in an intensive care unit as attention may be focused on more immediate problems associated with cardiac and respiratory function. The apparent onset of symptoms some days after operation may confuse the diagnosis, but such patients are often heavily sedated and amnesic, or if not, have more important discomforts which may mask symptoms in an extremity.

It may be suggested that a cluster of such cases may indicate some domestic vagary of operative or anaesthetic technique. Only two of the five cases were operated upon in Cardiff, the others at different centres elsewhere. The surgeon involved in case 3 agreed that exposure had been unusually wide and prolonged, but no anomalies emerged from enquiry concerning the others.

\section{References}

1 Po BJ, Hansen HR, Iatrogenic brachial plexus injury: a survey of the literature and of pertinent cases. Anesth Analg (Cleve) 1969; 48:915-22.

2 Davis DH, Onofrio BM, MacCarty CM. Brachial plexus injuries. Mayo Clin Proc 1978; 53:799-807.

3 Kwaan JHM, Rappaport I. Postoperative brachial plexus palsy. Arch Surg 1970; 101:612-5.

4 Stephens JW. Neurological sequelae of congenital heart surgery. Arch Neurol 1962; 7:450-9.

5 Kirsh MM, Magee KR, Gago O, Kahn DR, Sloan $\mathrm{H}$. Brachial plexus injury following median sternotomy incision. Ann Thorac Surg 1971; 11: 315-9.

6 Parks BJ. Postoperative peripheral neuropathies. Surgery 1973; 74:348-57.

7 Keates JRW, Innocenti DM, Ross DN. Mononeuritis multiplex. A complication of open-heart surgery. J Thorac Cardiovasc Surg 1975; 69: 816-9.

8 Honet JC, Raikes JA, Kantrowitz A, Pursel SE, Rubenfire M. Neuropathy in the upper extremity after open-heart surgery. Arch Phys Med Rehabil 1976; 57:264-7.

9 Jackson L, Keats AS. Mechanism of brachial plexus palsy. Anesthesiology 1965; 26:190-4. 